Bulletin d'Histoire Contemporaine de l'Espagne

$51 \mid 2017$

Les forces politiques durant la Seconde République espagnole

\title{
Le Movimiento ibérico de Liberación (MIL) et ses représentations dans la presse : mythes et mystifications
}

\section{Canela Llecha Llop}

\section{(2) OpenEdition}

\section{Journals}

Édition électronique

URL : http://journals.openedition.org/bhce/787

DOI : $10.4000 /$ bhce. 787

ISSN : 1968-3723

Éditeur

Presses Universitaires de Provence

Édition imprimée

Date de publication : 1 juin 2017

Pagination : 301-306

ISSN : 0987-4135

Référence électronique

Canela Llecha Llop, « Le Movimiento ibérico de Liberación (MIL) et ses représentations dans la presse : mythes et mystifications », Bulletin d'Histoire Contemporaine de l'Espagne [En ligne], 51 | 2017, mis en ligne le 09 octobre 2018, consulté le 23 septembre 2020. URL : http://journals.openedition.org/bhce/ 787 ; DOI : https://doi.org/10.4000/bhce.787 
encargándose en solitario de mantener los contactos con los estadounidenses durante las décadas de 1960 y 1970. Por este motivo (aunque no exclusivamente), durante aquellos años se produjo un progresivo descenso de los contactos entre los dirigentes vascos y los agentes del Departamento de Estado. Una reducción motivada por las sucesivas prórrogas de diez y cinco años a los convenios de 1953 , que en 1970 acabó provocando la ruptura de relaciones, a raíz de la firma de un nuevo acuerdo bilateral entre el Gobierno de los Estados Unidos y el de la España franquista.

A partir de entonces, los directores de la política vasca dejaron de colaborar con un Gobierno que, para ellos, socavaba las iniciativas a favor de la democracia y que con sus medidas políticas sostenía al régimen franquista. De este modo, Beitia reconvirtió la estrategia política atlantista, centrándose sólo en aquellos simpatizantes de la causa vasca situados en el Congreso y en los medios de comunicación, para presionar al Gobierno de Estados Unidos y que este, a su vez, condicionara los acuerdos que firmara con el Gobierno español al establecimiento de medidas liberalizadoras, que llevaran a España hacia un sistema democrático. Con todo, no fue hasta mediados de la década de 1970, cuando las cosas cambiaron en un sentido positivo, aunque en parte fuera motivado por la muerte de Franco en diciembre de 1975. Al año siguiente, las labores de lobbying de Beitia comenzaron a obtener victorias de especial notoriedad, consiguiendo -entre otras cosas- que sus «amigos del Capitolio» condicionaran la renovación de los acuerdos de ayuda mutua y de defensa (convertidos en Tratado de Amistad y Cooperación) a la implementación de medidas democratizadoras en el aparato institucional del Estado español. Sin embargo, para entonces, la rehabilitación de las instituciones democráticas en el País Vasco era un objetivo completamente real y alcanzable.

\section{Le Movimiento Ibérico de Liberación (MIL) et ses représentations dans la presse : mythes et mystifications ${ }^{1}$}

\section{Canela LLECHA LLOP}

Le Movimiento Ibérico de Liberación (MIL) fait partie de ces groupes politiques qui surgissent dans les années soixantedix et qui, refusant de se limiter à des objectifs antifranquistes, portent un projet révolutionnaire imprégné d'une conception internationaliste de la lutte. Ce travail de recherche cherche à approcher ce petit groupe anticapitaliste essentiellement au travers de ses représentations médiatiques. Pour ce faire, la thèse privilégie une approche double, qui croise perspective diachronique - un parcours de la presse sous le franquisme jusqu'aux médias en démocratie - et regard synchronique - une étude conjointe et comparative de différents journaux. L'examen du discours de presse relatif au MIL présente en effet un intérêt méthodologique majeur, dans la mesure où il fait apparaître une constellation d'ethos qui tient lieu d'identité politique au MIL. Les procédures de nomination ou de qualification du groupe, si elles en disent long sur les différentes configurations discursives dont elles émanent, contribuent à donner du MIL un portrait au kaléidoscope, qui cristallise toujours les langages et les problématiques contemporaines. En confrontant les textes du groupe lui-même à ceux des médias, la thèse aborde ce qui advient dans les jeux d'écart, de distorsion ou de recouvrement que cette mise en regard fait apparaître. Il s'agit d'étudier ce qui se joue au cœur de ces dispositifs rhétoriques, pour comprendre

1 Thèse d'Études Hispaniques en cotutelle soutenue le 12 décembre 2014 à l'Université Paris Ouest Nanterre La Défense, devant un jury composé de Mmes et MM. les Professeurs Bénédicte Brémard, Marie-Claude Chaput (directrice) Francis Demier, Géraldine Galeote, Pilar Martinez Vasseur, Julio Pérez Serrano (directeur). 
ce que les médias font au MIL et au projet politique dont il était porteur.

Les hypothèses de départ qui président à cette étude pourraient être formulées ainsi: bien que les médias écrits aient indéniablement contribué à faire connaître le MIL, ils ont systématiquement déformé ou passé sous silence l'idéal révolutionnaire défendu par le groupe, soit en dépolitisant son action, soit en utilisant politiquement l'histoire ou la mémoire du groupe pour établir, affirmer, ou renforcer des narrations exogènes. Ces procédés de mystification aboutiraient à une disqualification de facto de l'option politique défendue par le MIL, et, partant, à une restriction du champ des possibles idéologiques. L'analyse du cas du traitement du MIL par la presse dominante depuis une perspective diachronique devait permettre de mettre en évidence quels sont les présupposés catégoriels sous-jacents dans les débats propres à chaque moment historique. En ce sens, elle devait non seulement montrer par l'exemple la radicale historicité de toute interprétation, mais également élucider les mécanismes de construction d'une mémoire idéalisée, mythifiée ou mystifiée, du MIL. L'hypothèse de départ est que tout pouvoir, y compris celui à l'œuvre en démocratie, cherche à imposer l'univocité de sa narration canonique et qu'il a besoin pour ce faire de délégitimer ou d'absorber un certain nombre de narrations parallèles.

Pour éprouver ces hypothèses, la thèse adopte une structure tripartite.

Afin d'apprécier à quel point les récits que la presse véhicule sur le MIL sont mystifiants ou mythifiants, il fallait revenir en premier lieu sur le MIL lui-même. La première partie s'attache ainsi à présenter le contexte de parution du MIL en le replaçant dans le cadre national et international, le but étant d'historiciser les discours qu'il produit. La naissance d'un groupe comme le MIL est indissociable de la forte activité qui caractérise les « années 68 » au niveau international. Le contexte de la guerre froide et notamment de l'après-68 est marqué par le surgissement de nouvelles formations politiques, par de nouvelles revendications, mais également par un intense débat au sein de la gauche sur les modes d'action à adopter au moment même où le modèle de l'État providence semble entrer en crise, et dévoiler pleinement son rôle normatif. Se développent un certain nombre de groupes révolutionnaires qui, n'aspirant plus à conquérir le pouvoir, font de l'État et de la violence qu'il représente la cible privilégiée de leur lutte. Si l'Espagne est souvent présentée comme un cas à part dans le monde occidental, les problématiques qui lui sont propres sont toutefois intimement liées à la situation internationale. Au cours des années soixante, d'importantes transformations sociales et économiques se produisent, qui font miroiter à l'Espagne une intégration dans la Communauté Économique Européenne, et qui relancent par ailleurs l'organisation syndicale, tandis que l'opposition au franquisme s'organise, malgré une répression toujours féroce. C'est précisément dans ce contexte que le MIL fait son apparition à Barcelone à la fin des années soixante. Inextricablement relié au développement des luttes ouvrières qui se succèdent dans la capitale catalane, il hérite à la fois d'une composante libertaire certaine, mais s'inscrit également dans la filiation des nouveaux mouvements internationaux. L'analyse de la production du MIL, notamment des deux numéros de la revue C.I.A. Conspiración Internacional Anarquista, et des publications qu'il effectue au travers de la maison d'édition «Ediciones Mayo-37», a permis d'en produire une définition précise sur le plan politique. Point de confluence de diverses trajectoires vitales, le MIL a pour signes distinctifs un anti-autoritarisme et un anti-dirigisme notoires, qui font de lui un groupe hétérodoxe au niveau théorique se nourrissant de multiples influences et 
évoluant au gré des discussions et des événements. À travers la réactualisation de textes de penseurs hétérodoxes, le MIL s'emploie à critiquer les formations de la gauche classique (notamment le PC) qu'il juge réformistes, les groupes léninistes, maoïstes et marxistes-léninistes pour leur caractère dirigiste et, enfin, les formations trotskistes parce que trop déconnectées du mouvement ouvrier. En contrepoint, le MIL défend l'autonomie et l'autoorganisation de la classe ouvrière. Dès lors, il s'assigne un double rôle. D'une part, réaliser des actions d' " agitation armée» pour prouver que le mouvement ouvrier peut accroître le niveau de violence qu'il exerce, démontrant par là son potentiel révolutionnaire. D'autre part, développer le projet de «bibliothèque socialiste » qui a également pour but de provoquer l'agitation par l'auto-compréhension du mouvement révolutionnaire. Politiquement, le MIL peut être défini comme un groupe antiautoritaire et anti-léniniste qui reçoit les influences de mai 68 , notamment les idées conseillistes ou celles de l'Internationale Situationniste, et qui défend un anticapitalisme internationaliste révolutionnaire.

La deuxième partie de ce travail aborde plus précisément les modes de saisie du MIL, en prenant notamment en compte la concomitance entre les faits et leur mise en récit journalistique. Lors des dernières années du franquisme, le MIL réalise ses actions les plus retentissantes, durant une période de répression farouche. L'arrestation de Salvador Puig Antich et son exécution le 2 mars 1974 constituent ainsi des temps forts que la presse représentera dans les limites que permet le cadre du régime. Il est indéniable que le contrôle étroit des moyens de communication, et notamment de l'écrit, que continue d'exercer le régime explique très largement les narrations dépolitisantes dont fait l'objet le MIL pendant les dernières années du franquisme. En effet, « délinquants », « bandits », " assassins » et « anarchistes» sont les termes les plus récurrents qu'emploie la presse pour se référer aux membres du groupe. Ils s'inscrivent dans ce que l'on pourrait appeler une «tradition » ou un « habitus » rhétorique et herméneutique développés dès la fin de la Guerre civile et jusqu'aux derniers jours de vie d'un régime qui s'emploie à classer, pour mieux les dominer, tous les mouvements de contestation sous des appellations moralement et juridiquement accablantes. La référence à la violence politique qualifiée de terroriste, ou l'inscription de Puig Antich, et à travers lui de l'ensemble du MIL, dans la seule famille anarchiste, sont autant de catégories d'appréhension qui, bien que resémantisées et réinvesties à l'aune des problématiques contemporaines, reviennent dans la presse de façon répétée, et ce bien après la fin du régime qui les a bâties.

Avec le processus de Transition des années 1974-1979, et le début de la libéralisation de l'accès à l'information, la référence au MIL ou à ses membres croît sensiblement dans la presse. Tout d'abord, le traitement que subit dans la presse la violence politique à laquelle est associé le MIL de façon récurrente se transforme. Le discours médiatique développe, rejoignant ainsi la rhétorique qui a cours dans la presse des démocraties européennes voisines, un cadre d'interprétation binaire fondé sur l'opposition entre terrorisme et antiterrorisme. Dans le cas espagnol cependant, la désignation pendant la Transition de tout acte violent comme ressortissant au «terrorisme extrémiste » a permis de discréditer politiquement les options critiques du processus démocratique en marche. Mais la saisie du MIL est également adossée à certains des mouvements revendicatifs les plus largement investis par la société civile, qu'il s'agisse de réclamer une véritable amnistie pour les prisonniers politiques ou de défendre la nécessité de reconnaître 
la liberté d'expression pour construire une véritable démocratie. L'exemple de la représentation du MIL montre à quel point la période transitionnelle est à la fois un moment de résurgence d'un certain nombre de problématiques conflictuelles que quarante ans de franquisme ont éclipsées, mais aussi le temps de leur " désamorçage » par intégration dans le jeu parlementaire. Malgré une volonté affichée d'inclusion universelle, et malgré la conquête de droits fondamentaux qu'elle permet, cette démocratie «constitutionnaliste» produit toutefois des mécanismes d'oblitération (question de la forme de l'État, question de la participation citoyenne, etc.). Ainsi, la prise en charge depuis l'État des questions épineuses soulevées pendant cette période de transition par le biais de leur judiciarisation dénote une volonté de pacification de la société civile - ce que cristallise parfaitement le terme de " consensus » tant invoqué pour se référer à ce moment historique.

Si la présence médiatique du MIL ou de ses membres est particulièrement importante dans les années 1974-1980, à première vue, la période comprise entre la fin des années soixante-dix et le milieu des années quatre-vingt-dix semblerait correspondre à une manière de "traversée du désert ", les références au groupe et à ses membres se faisant rares. Cependant, à y regarder de plus près, c'est une véritable mutation de la nature des informations concernant le MIL, concomitante de leur redéploiement de la sphère du politique vers celle du culturel ou du sociétal, qui se produit. Le mécanisme, propre à la presse, de recherche éperdue de la nouveauté, explique que l'abandon progressif de la référence au MIL s'accompagne d'une forte personnalisation de la représentation: Puig Antich devient le seul membre du groupe à avoir droit de cité dans les journaux. Ces phénomènes d'individualisation de l'information et de diversification des rubriques de parution des articles concernant le MIL/Puig Antich témoignent de l'émergence de nouvelles grilles interprétatives appliquées au groupe: tantôt événement historique, tantôt repère temporel, le syntagme devient une référence culturelle et rapidement une figure mémorielle. Cependant, si Puig Antich continue d'être cité régulièrement dans la presse, il n'apparaît que de façon oblique, en tant que prétexte ou point de départ pour aborder des problématiques exogènes, comme celle du régionalisme catalan. D'autre part, son nom est toujours associé à l'anarchisme, ici réinvesti dans un sens bien plus positif, et intégrant la famille très floue de l'« antifranquisme» dont la narration commence à être élaborée dans ces annéeslà. Finalement, dans le prolongement de ce mouvement, c'est à la question mémorielle (qui commence à faire l'objet de revendications dans certains secteurs de la société) que le nom de Puig Antich se retrouve associé. Dans tous les cas, qu'il y ait « usage politicien», «culturalisation» ou «mémorialisation» de la figure de Puig Antich - et, partant, du MIL -, c'est tout un pan de la charge politique qu'elle portait initialement qui se trouve invariablement oblitéré, voire évacué. Plus largement, ce qu'illustre l'évolution que connaissent les catégories interprétatives appliquées au MIL, c'est un changement de paradigme majeur, la référence révolutionnaire étant, en Espagne comme dans l'ensemble des démocraties occidentales, abandonnée au profit de celle de la réforme.

Dans la troisième partie, la thèse analyse comment la naissance et le développement du «mouvement pour la récupération de la mémoire historique» étaient symptomatiques de la promotion d'un paradigme mémoriel à l'aune duquel va désormais être appréhendé le MIL. En Espagne, initialement emmenée par les "mouvements pour la récupération de la mémoire », la question mémorielle gagne la sphère politique et devient un 
argument fort dans la bataille que se livrent les partis aspirant à gouverner. La presse se fait ainsi largement l'écho des polémiques que suscitent, entre autres, les discussions parlementaires sur la «Ley por la que se reconocen y amplianderechos y se establecenmedidas en favor de quienes padecieron persecución o violencia durante la guerra civil y la dictadura », communément appelée "Ley de memoria histórica », finalement approuvée en décembre 2007. Ainsi, en quelques années, la question mémorielle est venue saturer le débat sociétal, culturel, politique, et médiatique. Partant de cette prééminence du débat sur le passé récent de l'Espagne, l'objectif de cette partie était de montrer comment s'élabore, notamment dans les médias, la construction de la mémoire sur le MIL.

En peu de temps, la figure de Puig Antich est érigée en exemple paradigmatique des revendications pour la révision des procès du franquisme, la presse suivant pratiquement sur le mode de la chronique l'évolution de la demande de révision de son procès intentée par ses sœurs. Devenant la métonymie de ces demandes, son nom est bien souvent utilisé pour montrer les limites de la "Loi de Mémoire Historique». L'analyse de ce procédé de métonymisation m'a permis de comprendre comment la question mémorielle se judiciarisait. La catégorie de "victime» étant désormais systématiquement appliquée à Puig Antich, elle indique que cette "récupération» s'effectue dans les limites d'une appréhension juridique de sa personne. Mais cet usage en tant qu'arme de la lutte mémorielle induit également une réduction de Puig Antich à un certain nombre de traits qui font de lui une figure maniable, parce que stéréotypée. J'ai ainsi par la suite étudié les différentes nominations dont il a fait l'objet, et les processus de construction de mémoires concurrentes que les différents qualificatifs qui lui sont apposés signifient.
Qualifié d' « anarchiste» et de "catalan », c'est finalement à la famille antifranquiste qu'il est invariablement associé, Puig Antich étant progressivement défini comme combattant catalan antifranquiste victime du régime. Cette catégorisation est le résultat simultané de la judiciarisation de la «récupération» de la mémoire, de l'élaboration d'un récit fondateur de la démocratie basé sur les luttes antifranquistes, et partant, de la construction d'une légitimité supplémentaire pour les institutions catalanes qui entretiennent un bras-de-fer avec le Gouvernement central. Ainsi, la thèse montre dans quelle mesure les usages publics de la figure de Puig Antich ont été à bien des égards intéressés. Plus largement, l'exemple des représentations et des nominations de Puig Antich dans la presse a conduit à envisager le caractère profondément excluant du processus de la « récupération » de la mémoire.

Enfin, le film Salvador de Manuel Huerga, et la polémique que sa sortie en 2006 a suscitée, cristallisent le débat mémoriel qui a cours dans la sphère sociétale et politique. L'étude de ce film a permis en outre d'éclairer les difficultés d'une construction hétérodoxe de la mémoire: si le film prétend réhabiliter la mémoire de Puig Antich que le processus transitionnel aurait oblitérée, c'est in fine la mémoire antifranquiste qui est revalorisée, les spécificités de Puig Antich, et donc du MIL, étant soumises à l'impératif de cette réhabilitation. La récupération dans et par la mémoire consensuelle de Puig Antich en tant que martyr romantique de l'antifranquisme étouffe le débat public qu'une problématisation historique de ses choix idéologiques pourrait susciter. Le film de Huerga est polémique et problématique dans la mesure où il prétend remettre sur le devant de la scène la brutalité de la répression franquiste, mais cette volonté de combattre l'oubli produit à son tour de l'exclusion. 
Ces « oublis » de la mémoire consensuelle nous parlent aussi dans une certaine mesure du présent: ils définissent, en creux, ce qui peut et ce qui ne peut pas être « récupéré », « remémoré ». C'est donc la bataille pour la construction de la mémoire de la fin du franquisme et du processus de la Transition qui apparaît en filigrane, et par conséquent, la potentielle remise en cause du pacte fondateur de la démocratie espagnole. C'est parce que la "récupération» de la mémoire a lieu depuis le présent, et de façon bien souvent présentiste, que les fondements de la démocratie sont l'enjeu majeur de la lutte et des polémiques qui ont cours. Si d'aucuns refusent obstinément de faire retour sur le passé, considérant que le processus transitionnel l'a adéquatement clos, le mouvement pour la « récupération » de la mémoire tente, pour sa part, de rééquilibrer ce qui est ressenti comme un tort historique en construisant une narration dans laquelle les luttes de l'antifranquisme viendraient légitimer la démocratie. Si le régime sortant a assuré - notamment au travers de la figure du Roi - la légalité $\mathrm{du}$ processus transitionnel, ce serait l'opposition antifranquiste qui lui fournirait une légitimité. Dans les deux cas, c'est la démocratie telle qu'elle a été construite depuis le pacte transitionnel qui apparaît comme le référent ultime: envisagé depuis le présent démocratique, le passé est ainsi relu téléologiquement. C'est parce que le paradigme mémoriel dominant dans les dernières années se construit en prenant comme point de fuite idéal le présent démocratique que l'espace discursif occupé par les expériences militantes hétérodoxes est restreint à l'extrême.

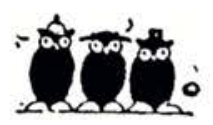

Genre et classe : poétiques gay dans l'espace public de l'Espagne postfranquiste (1970-1988) ${ }^{1}$

\section{Brice Chamouleau de MATHA}

Pourquoi, si la démocratisation de l'Espagne après la dictature franquiste est exemplaire, les archives judiciaires font parfois état d'une répression contre des subjectivités homosexuelles jusque dans la deuxième moitié des années 1980 à Barcelone? L'étude des archives des tribunaux de Dangerosité et de Réhabilitation Sociale franquistes, à Barcelone, permet de mettre en tension un récit que l'Espagne actuelle partage avec d'autres démocraties occidentales sur l'inclusion politique des minorités sexuelles. En Espagne, cette inclusion est symptomatique du bon ordonnancement démocratique après la dictature: si le dictateur Franco réprimait les subjectivités homosexuelles avec la Loi de Dangerosité Sociale votée en 1970, la démocratie les reconnaît progressivement jusqu'à en faire les sujets de la rédemption démocratique. Elles incarnent le chemin vers la liberté entrepris par l'Espagne depuis 1975 d'après le président Zapatero lorsqu'est votée la loi sur le mariage homosexuel. Le mariage gay et la loi de 2007 sur le changement de sexe civil qui n'exige plus d'avoir subi une opération des organes sexuels sont les deux piliers qui constituent le point d'arrivée de cette narration collective qui s'enracine dans la transition démocratique, au moment où les Espagnols plébiscitent la Constitution de 1978 et qu'est abrogé l'article pénalisant les « actes d'homosexualité ».

1 Thèse soutenue à l'Université de Bordeaux III, le 24 novembre 2014 , devant un jury composé de $\mathrm{M}^{\text {mes }}$ et $\mathrm{MM}$. les profs. Marie Franco (Univ. de Paris III, rapporteur), François Godicheau (Univ, de Bordeaux III, directeur), Jesús Izquierdo Marín (Univ. Autónoma, Madrid), Aránzazu Sarria Buil (Univ, de Bordeaux III) et Mercedes Yusta (Univ, de Paris VIII, présidente). 